\title{
Danger of the era: environmental obesogens
}

\begin{abstract}
Obesity is a global health problem in recent years. Adipose tissue was known as a storage tissue increasing with rise of the body mass index the indicator of obesity. Recently, adipose tissue is well known to be an endocrine organ with endocrine and metabolic functions. Interruption of endocrine functions of adipose tissue might contribute to obesity. Many environmental chemicals affect actions of hormones so these chemicals have been defined as endocrine-disrupting chemicals (EDCs) or endocrine disruptors. With the increase in production of EDCs in the world parallels the global obesity prevalence increase. These chemicals commonly found in pesticides /herbicides, industrial and household products, plastics detergents and personal care products. Humans are mainly exposed to EDCs with contaminated water and food, particularly with meat, fish and dairy products as animals also accumulate EDCs in their adipose tissue; dermal absorbtion as well as inhalation of polluted air. The reported rise in obesity of children under 2 years of age is also suggestive of alterations during development. In fetal and early life EDCs exposure may also have an influence on epigenetic programming of obesity. These pollutants can pass from mother to fetus via placenta and breast feeding. EDCs are also thought to alter microbiata profile thus resulting in obesity as well as altering related epigenetic variations. Studies also report that gender, dose, life stage and duration of EDCs exposure are also important factors in altered functions, but it is still not clear that which chemical at which dose and duration may cause the significant alterations. Some obesogens are found in nature such as phytoestrogens and the others are mostly synthetic chemicals. These substances are defined persistant organic pollutant (POPs) and they accumulate in adipose tissue as they ara lipophilic chemicals. Common EDSs are Tributyltin (TBT), 4-Nonylphenol used in industry, persistent organochlorines (POPs) used in agriculture industry, diethylstilboestrol (DES) as a pharmaceutical, bisphenol A (BPA) and phthalates used in plastics, parabens as preservatives, phytoestrogens in edible plants, polybrominated diphenylethers (PBDEs) as flame retardants. We aimed to overview common environmental pollutants accepted as EDCs with their sources humans are exposed in daily life, their health contributions and acting mechanisms in general and searched current literature with the key words: obesogens, POPs, endocrine disruptors in pubMed and filtered the results with articles in recent 5 years. One of the main issues of the world is obesity which is related to genetic and environmental factors. Although obesity is related with excessive food consumption or lack of exercise with the increasing industrialization it is also strongly associated with environmental factors such as synthetic chemicals and pollutants. Although there are policies to reduce the EDCs exposere, individual precautions and conscious consumers tought to be more effective. This mini review provides current experience about association between obesity and endocrine disrupting chemicals.
\end{abstract}

Keywords: Obesity, Endocrin disrupting chemicals, Environmental pollutants
Mini Review

Volume 8 Issue I - 2018

Açelya GÜL, Binnur Okan Bakır

Yeditepe University, Department of Nutrition and Dietetic

Correspondence: Binnur OKAN BAKIR, Yeditepe University, Department of Nutrition and Dietetic, Tel +90 021657800-1657, Email binnur.bakir@yeditepe.edu.tr

Received: December 15, 2017 | Published: February 27, 2018
Abbreviatıons: PPARS, Peroxisome proliferator activated receptors; EDCs, Endocrine disrupting chemicals; POPs, Persistant organic pollutant; TBT:Tributytin; DES, Diethylstilbestrol; BPA, Bisphenol A; BDEss, Polybrominated diphenylethers; DDT, Dikloro difenil trikloroethan; DDE, Dikloro difenil dikloroetilen.

\section{Adipose tissue functions and alterations due to EDCs}

Obesity is a global health problem in recent years. ${ }^{1,2}$ Adipose tissue was known as a fat storage tissue increasing with the rise of the body mass index the indicator of obesity. Recently it is well known that adipose tissue is not only a storage tissue but also has endocrine and metabolic functions. ${ }^{3,4}$ The major body weight regulators and inflammatory agents such as leptin, peroxisome proliferator-activated receptors (PPARs) is controlled by adipose tissue.These regulators stimulate food intake, metabolic efficiency, and energy storage. ${ }^{4,5}$ Interruptions in endocrine functions of adipose tissue may contribute to obesity. ${ }^{6}$ Many environmental chemicals, are mainly lipophilic and accumulate in adipose tissue, affect actions of hormones so these chemicals have been defined as endocrine-disrupting chemicals(EDCs) or endocrine disruptors. ${ }^{1,7}$ With the increase in production of EDCs in the world parallels the global obesity prevalence increases. ${ }^{8,9}$ Obesogens as xenobiotic chemicals which bring about decreasing detoxification mechanism of body may alter the normal progress of adipogenesis or energy balance. . $, 8,10,11^{2}$

\section{EDC contamination}

EDCs occur at all parts of the environment as they are present in surface and ground water, in rivers, lakes, oceans; in precipitates, 
lands and plants. ${ }^{12,13}$ They appear as they transfer to land via air and back to air when their concentrations in the air are reduced as well as their circulation between water and air and/or lands seems to be the same. ${ }^{13,14}$ These chemicals commonly found in pesticides /herbicides, industrial and household products, plastics detergents and personal care products. ${ }^{1,7}$ These synthetic chemicals are enduring so they do not degrade in the nature. For this reason these substances are defined persistant organic pollutant(POPs) and they accumulate in adipose tissue. ${ }^{15,16}$ Some obesogens are found in nature such as phytoestrogens and the others are mostly synthetic chemicals. Humans are mainly exposed to EDCs with contaminated water and food, particularly with meat, fish and dairy products as animals also accumulate EDCs in their adipose tissue; dermal absorbtion as well as inhalation of polluted air. ${ }^{8}$ They are lipophilic and bioaccumulate in the food chain and human body. More obese the individual is more chemicals are accumulated and more accumulated chemicals induces more fat deposit as they are endocrine disruptors and damages the endocrine control of body weight. ${ }^{1}$

\section{Common EDCs}

Widespread EDCs which human are known to be exposed are Tributyltin (TBT), 4-Nonylphenol used in industry, persistent organochlorines (POPs) used in agriculture industry, diethylstilboestrol (DES) as a pharmaceutical, bisphenol A (BPA) and phthalates used in plastics, parabens as preservatives, phytoestrogens in edible plants, polybrominated diphenylethers (PBDEs) as flame retardants. Tributyltin(TBT) used as a biocide in antifouling paints applied to the hulls of ships and cause imposex in molluscs and to masculines female fish. ${ }^{1,17}$ TBT may inhibit aromatase which is responsible for the conversion of testosterone into estrogens. ${ }^{1,18,19}$ 4-Nonylphenol is has eustrogenic effets and used as a surfactan in industry and domestic applicarion. ${ }^{1,20,21}$ POPs are used mostly as insecticides (DDT, DDE) and Diethylstilbestrol (DES) is a non steroidal estrogen. The DES toxicity includes its interference with reproductive system and association with female reproductive tract cancers. ${ }^{1,22}$ Bisphenol A a componenet of polycarbonat plastics and resins. It is ubiquitous in food and beverage containers, baby bottles, toys, dental sealants. Foods and beverage can be contaminated by BPA as a result of heating or acidic/basic condition during storage. ${ }^{1,8}$ Phythalates are diester of phthalic acid .They are used in commonly plastic scuh as polyvinyl chloride and some products such as food and beverage packaging, soaps, shampoos,cosmetics and hair sprays. ${ }^{8}$ Parabens are mostly found in personal care products, foods, pharmaceutical products and paper products. ${ }^{1,23}$ Phytoestrogens are found naturally in plant such as soy and soy products. They are defined dietary estrogens. Increased consumption of soy products and soy supplements cause exposure to phytoestrogens. Genstein which is predominant isoflavone in soybean is responsible of changes in the metabolic and adipogenic regulators such as PPAR $y \cdot{ }^{8,24,25}$ Recent experimental data supported that genistein dysregulates the body composition, in a dose-dependent and genderspecific manner, disrupting and reprogramming the signals dictating adipose tissue expansion, likely throughout the early-life epigenetic regulation of Wnt10b, a further key adipogenic gene in adipose tissue. ${ }^{8,26}$ Polychlorinated Bisphenols are organic chemicals and found in electricam equipment. Some PBCs are identified as EDCs because of their estrogenic and antiandrogenic effects. ${ }^{8,27}$ Although PCBs were banned at the end of the 1970s in many countries because of environmental toxicity, these compounds still remain present in the environment due to their high stability and represent one of the key constituents of POPs all over the world. ${ }^{8,28}$ Perfluoroalkyl substances (PFASs) are non organochlorine POPs used in industrial applications. PFASs are detectable in human blood. ${ }^{8}$ Pesticides consumption is the most important source of EDCs exposure. ${ }^{1,29}$ Two main types of pesticided are organochlorine and organophosphate pesticides. Although Organochlorine pesticides was banned, they are still detected in tap water. Organophosphate pesticides have replaced organochlorine. ${ }^{1,29}$ These pesticides enter into food chain and tends to persist environment and accumulate human body. ${ }^{8,30}$

\section{Acting mechanisms of EDCs on human body}

Human body might be exposed to these chemicals with oral intake by contaminated water and food consumption, respiration, or dermal absorption. ${ }^{1,7}$ EDCs related to obesity may involve in several mechanisms. EDCs distrupt lipid and glucose metabolism (endocrine regulated metabolic process) and also neuroendocrine control of appetite and satiety. ${ }^{1,9,17,31,32}$ Moreover EDCs affect pro-inflammatory mechanisms by activating oxidative stress- sensitive transcription factors such as nuclear factor kappa B (NFKB) stimulating cytokines, chemokines and adhesion molecules in the vascular endothelium. Along the pro-inflamatory mechanisms, EDCs are associated with an increased risk of non communicable or chronic diseases such as cardivascular diseases or diabetes and insulin resistance. , $^{1,18,33,34}$ As EDCs are accumulated in fat tissue several studies shown an increasing in blood POPs following fat loss when obese individuals lose weight with diet or bariatric surgery, with approximately $30 \mathrm{~kg}$ decrease in fat Mass. ${ }^{3,35,36}$ Gender dose, life stage and duration of EDCs exposure are also important factors. ${ }^{1,37}$ In obesogen hypothesis, EDCs effect obesity related epigenetic variations and microbiata profile. ${ }^{8,38}$ EDCs stimulate expression of noncoding RNAs, changes in chromatin structure and DNA and histone methylation. ${ }^{8}$ Low levels of DNA methylation can produce active adipogenic genes so adipocyte number increases. Increasing active adipogenic genes may change metabolic set point. ${ }^{8,38}$ According to set point theory, body weight sustains stable from internal regulatory mechanisms that are genetically determined. ${ }^{39}$ Change in metabolic set point causes weight regain after weight loss. ${ }^{8,38}$

In $14^{\text {th }}$ week of gestation, adipogenesis begins ${ }^{8,40}$ and goes on during postnatal period. ${ }^{6,41}$ The reported rise in obesity of children under 2 years of age is also suggestive of alterations during development. ${ }^{1,42,43}$ In a study with children in the Faroe Island shown that prenatal exposure to polychlorinated bisphenols and dikloro difenil dikloroetilen contained in seafood is related with increased body weight. ${ }^{1,44}$ In early life EDCs exposure can influence epigenetic programming of obesity. ${ }^{1}$ These pollutants can pass from mother to fetus via placenta and breast feeding. In a cohort conducted in 189 adult obese individuals aimed evaluation of POPs level in visceral and subcutaneous adipose tissue has found that both were affected with POPs but visceral adipose tissue was more sensitive to accumulation. Additionally they found a relationship between increased POPs accumulation and increased metabolic risk as dysglycemia and high blood pressure had positive relationhip with elevated POPs level.

A rodent model experiment female mice were fed with high fat and high sucrose diet with or without pollutants. The pollutants were prepared as a mixture of low doses. It contained 'Tolerable Daily Intake(TDI)' doses of tetrachlorodibenzo-p-dioxin, PCB, bisphenol A and phthalate. Each pollutant was used at a dose grossly corresponding to the TDI reference dose. They found that mice fed with pollutants 
even at TDI doses had increased body weight, plasma fasting glycose and plasma insulin levels. Additionally they have out that metabolic outcomes were sex and age depended that female were more sensitive to exposure in diffrerent stages of life span.

\section{Conclusion}

One of the main issues of the world is obesity which is related to genetic and environmental factors. Although obesity is related with excessive food consumption or lack of exercise with the increasing industrialization it is also associated with strongly environmental factors such as synthetic chemicals and pollutants. Common EDSs are Tributyltin (TBT), 4-Nonylphenol used in industry, persistent organochlorines (POPs) used in agriculture industry, diethylstilboestrol (DES) as a pharmaceutical, bisphenol A (BPA) and phthalates used in plastics, parabens as preservatives, phytoestrogens in edible plants, polybrominated diphenylethers (PBDEs) as flame retardants. While these chemicals are known to be used industrially, many chemicals may occur unintentionally during industrial processes. Obesogens as xenobiotic chemicals which bring about decreasing detoxification mechanism of body can alter the normal progress of adipogenesis or energy balance. Environmental pollutants and poor nutritional patterns affect hormonal regulation, metabolic pathways, cell modification/stress and thus resulting in increased disease risk due to long term effects of gene expression. In other words obesogenes causing alterations in PPARy activation and causes elevated fat cell quantity and volume which results in obese humans and animals.

When considering the relationship between obesity and EDCs, production of EDCs must be controlled by policies and exposure to these substances especialy in early life stages should be reduced. Two important policies are applied for EDCs exposure.The UNEP Stockholm Convention (UNEP, 2001) propose to eliminate or reduce the production and use of persistent organic pollutants. The other protocol The Aarhus Protocol on Persistent Organic Pollutants examines to eliminate any discharge, emissions and losses of persistent organic pollutant in Europe, some former Soviet Union conuntries and The United States. The Protocol bans the production and use of some products outright. Although there are policies to reduce the EDCs exposere, individual precautions and conscious consumers tought to be more effective.

\section{Recommendation}

Industrilization and processes in food supply are neccessary for global increase of human population and development. In order to reduce exposure to EDCs, personal cautions might be summarized as maintaining ideal body weight as individuals with more adipose tissue are vulnerable for more accumulation of EDCs.

Diet should include a variety of foods as potential harm from contaminants has to be compensated with potential benefits and also in order to decrease exposure to a spesific EDC from a spesific source. Animal sourced fats which are high in persistent toxics due to bioaccumulation should be decreased and before meat, fish and poultry consumption fat and skin should be trimmed. Vegetables and fruits should be washed thoroughly with a brush and fruits and vegetables which have any damage should not be selected and outer layer of the leafly vegetables should be peeled. For reduction of industrial chemical exposure it is important to be aware of the spesific EDC sources which are commonly in use during daily life such as cosmetic products, detergent, packaged food and plastics and should be considered during selection of the relevant product.

\section{Acknowledgment}

None.

\section{Conflicts of interest}

None.

\section{References}

1. Darbre PD. Endocrine and Disruptors and Obesity. Curr Obes Rep. 2017;6(1):18-27.

2. OECD. Obesity update. 2014.

3. Merril ML, Emond C, Kim MJ, et al. Toxicological Function of Adipose Tissue: Focus on Persistent Organic Pollutants. Envirronmental Health Perspectives. 2013;121:2.

4. Grün F, Blumberg B. Environmental Obesogens: Organotins and Endocrine Disrupion via Nuclear Receptor Signaling. Endocrinology. 2006;147(6):S50-S55.

5. Auwerx J. PPAR gama, the Ultimate Thrifty Gene. Diabetologia. 1999;42(9):1033-1049.

6. Janesick AS, Blumberg B. Obesogens: an Emerging Threat to Public Health. Ame J Obs Gynecol. 2016;214(5):559-565.

7. Darbre PD. Endocrine disruption and human health. New York: Academic; Overview of EDCs and human health which sets the bigger picture. Curr Obes Rep. 2017;6(1):18-27.

8. Nappi F, Barrea L, Somma CD, et al. Endocrine Aspects of Environmental "Obesogens" Pollutants. Int J Environ Res Public Health. 2016;13(8):765.

9. Gore AC, Chappell VA, Fenton SE, et al. EDC-2: The Endocrine Society's second scientific statement on endocrine-disrupting chemicals. Endocr Rev. 2015;36(6):E1-E150.

10. Barouki R. Linking long-term toxicity of xeno-chemicals with short-term biological adaptation. Biochimie. 2010;92(9):1222-1226.

11. Wang G, Ma P, Zhang Q, et al. Endocrine disrupting chemicals in New Orleans surface waters and Mississippi Sound sediments. J Environ Monit. 2012;14(5):1353-1364.

12. Annamalai J, Namasivayam V. Endocrine disrupting chemicals in the atmosphere: their effects on humans and wildlife. Environ Int 2015;76:7897.

13. Lohmann R, Breivik K, Dachs J, et al. Global fate of POPs: current and future research directions. Environ Pollut. 2007;150(1):150-165.

14. Ozonoff D, Aschengrau A, Coogan P. Cancer in the vicinity of a department of defense superfund site in Massachusetts. Toxicol Ind Health. 1994;10(3):119-141

15. Li QQ, Loganath A, Chong YS, et al. Persistent organic pollutants and adverse health effects in humans. $J$ Toxicol Environ Health $A$. 2006;69(21):1987-2005.

16. Pestana D, Faria G, Sa C, et al. Persistent Organic Pollutant Levels in Human Visceral and Subcutaneous Adipose tissue in Obese IndividualsDepot Differences and Dysmetabolism implications. Environ Res. 2014;133:170-177.

17. Horiguchi T. Masculinization of female gastropod mollusks induced by organotin compounds, focusing on mechanism of actions of tributyltin and triphenyltin for development of imposex. Environ Sci. 2006;13(2):77-87. 
18. Saitoh M, Yanase T, Morinaga H, et al. Tributyltin or triphenyltin inhibits aromatase activity in the human granulosa-like tumor cell line KGN Biochemical and biophysical research communications. 2001;289(1):198204.

19. Cooke GM. Effect of organotins on human aromatase activity in vitro Toxicol Lett. 2002;126(2):121-130.

20. OECD (Organisation for Economic Cooperation and Development) The 2004 OECD list of high production volume chemicals. Environment Directorate, Paris; 2004

21. White R, Jobling S,Hoare SA, etal.Environmentallypersistentalkylphenolic compounds are estrogenic. Endocrinology. 1994;135(1):175-182.

22. Koebnick C, Smith N, Coleman KJ, et al. Prevalence of extreme obesity in a multiethnic cohort of children and adolescents. $J$ Pediatr. 2010;157(1):26-31.

23. Darbre PD, Harvey PW. Parabens can enable hallmarks and characteristics of cancer in human breast epithelial cells: a review of the literature with reference to new exposure data and regulatory status. $J$ Appl Toxicol. 2014;34(9):925-938.

24. Patisaul HB, Jefferson W. The pros and cons of phytoestrogens. Front Neuroendocrinol. 2010;31(4):400-419.

25. Penza M, Montani C, Romani A, et al. Genistein affects adipose tissue deposition in a dose-dependent and gender-specific manner. Endocrinology. 2006;147(12): 5740-5751.

26. Strakovsky RS, Lezmi S, Flaws JA, et al. Genistein exposure during the early postnatal period favors the development of obesity in female, but not male rats. Toxicol Sci. 2014;138(1):161-174.

27. Portigal CL, Cowell SP, Fedoruk MN, et al. Polychlorinated biphenyls interfere with androgen-induced transcriptional activation and hormone binding. Toxicol Appl Pharmacol. 2002;179(3):185-194.

28. Ghosh S, Murinova L, Trnovec T, et al. Biomarkers linking PCB exposure and obesity. Curr Pharm Biotechnol 2014;15(11):1058-1068.

29. Slotkin TA. Does early-life exposure to organophosphate insecticides lead to prediabetes and obesity? Reprod Toxicol. 2011;31(3): 297-301.

30. Mostafalou S. Persistent organic pollutants and concern over the link with insulin resistance related metabolic diseases. Rev Environ Contam Toxicol. 2016;238:69-89.

31. Rubin BS. Bisphenol A: an endocrine disruptor with widespread exposure and multiple effects. J Steroid Biochem Mol Biol. 2011;127(1-2):27-34.
32. Report EUR17549 of the Environment and Climate Change Research Programme of DGXII of the European Commission. Weybridge, UK; 1996.

33. Kamrin MA. Phthalate risks, phthalate regulation and public health: a review. J Toxicol Environ Health Part B. 2009;12(2):157-174.

34. Bramwell L, Glinianaia SV, Rankin J, et al. Associations between human exposure to polybrominated diphenyl ether flame retardants via diet and indoor dust, and internal dose: a systematic review. Environ Int. 2016;92:680-694.

35. Hue O, Marcotte J, Berrigan F, et al. Increased plasma levels of toxic pollutants accompanying weight loss induced by hypocaloric diet or by bariatric surgery. Obes Surg. 2006;16(9):1145-1154.

36. Kim MJ, Marchand P, Henegar C, et al. Fate and complex pathogenic effects of dioxins and polychlorinated biphenyls in obese subjects before and after drastic weight loss. Environ Health Perspect. 2011;119(3):377383.

37. Cheikh Rouhou M, Karelis AD, St-Pierre DH, et al. Adverse effects of weight loss: Are persistent organic pollutants a potential culprit? Diabetes Metab. 2016;42(4):215-223.

38. Mahan LK, Escott-Stump S, Krause MV. Krause's food \& nutrition therapy. Elsevier Saunders; 2017. 385 p.

39. Poissonnet CM, Burdi AR, Garn SM. The chronology of adipose tissue appearance and distribution in the human fetus. Early Hum Dev. 1984;10(1-2):1-11.

40. Spalding KL, Arner E, Westermark PO, et al. Dynamics of fat cell turnover in humans. Nature. 2008;453(7196):783-787.

41. Kim J, Peterson KE, Scanlon KS, et al. Trends in overweight from 1980 through 2001 among preschool-aged children enrolled in a health maintenance organization. Obesity. 2006;14(7):1107-1112.

42. Tang-Péronard, JL, Heitmann BL, Andersen HR, et al. Association between prenatal polychlorinated biphenyl exposure and obesity development at ages 5 and $7 \mathrm{y}$ : a prospective cohort study of 656 children from the Faroe Islands. Am J Clin Nutr. 2014;99(1):5-13.

43. Naville D, Labaronne E, Vega N, E et. al. Metabolic Outcome of Female Mice Exposed to a Mixture of Low-Dose Pollutants in a Diet Induced Obesity Model. PloS One. 2015;10(4):e0124015.

44. Heindel JJ, vom Saal FS. Role of nutrition and environmental endocrine disrupting chemicals during the perinatal period on the aetiology of obesity. Mol Cell Endocrinol. 2009;304(1-2):90-96. 